\title{
Video
}

\section{RIGHT LAPAROSCOPIC ADRENALECTOMY FOR CONN'S SYNDROME}

\section{SUPRARRENALECTOMIA DERECHA LAPAROSCÓPICA POR SÍNDROME DE CONN}

Ana García-Vico ${ }^{1 *}$, Jesús Cañete-Gómez ${ }^{1}$, Jose Manuel Lorente-Herce ${ }^{1}$

${ }^{1}$ Servicio de Cirugía General y del Aparato Digestivo del Hospital Virgen de Valme, Sevilla.

*Corresponding Author:

Calle Carmen Martínez Sancho N05, E09 P5A Sevilla, 41008 Spain.

Email: anagarciavico91@gmail.com

Telephone: +34-645313975

Received: 19 February 2020, Approved: 06 April 2020, Published: June 2020

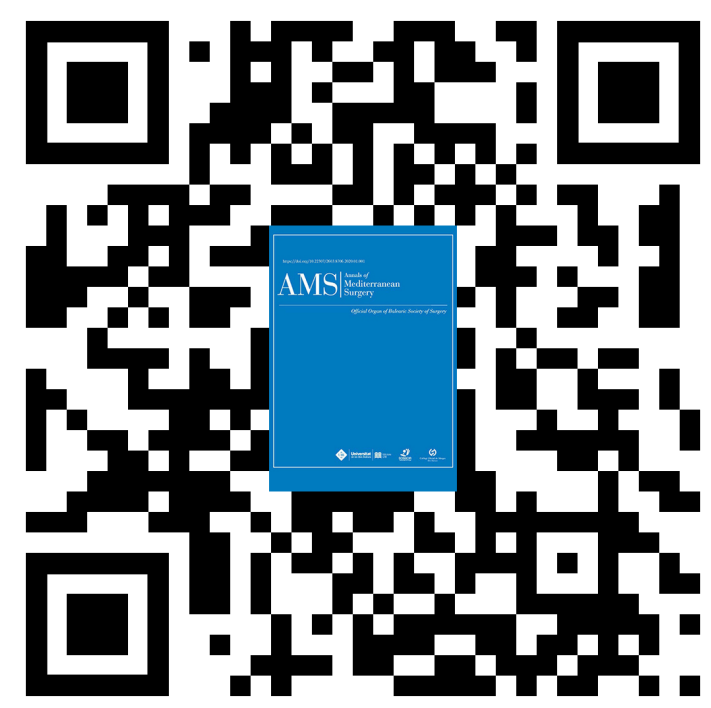

\section{Abstract}

Introduction: adrenal adenomas are benign tumors that may have hormone hiperproduction. Conn`s Syndrome is cause of secondary arterial hypertension due to excessive production of aldosterone conducting to primary hiperaldosteronism.

Clinical case: a 52-year-old female with a 4 year history of hypertension with poor control despite medication during the last 2 months. Secondary HTA was suspected son the patient was investiga-ted: High levels of aldosterone were found on blood analisis. The CT scan showed a right adrenal mass. Given this suspicion, a right laparoscopic adrenalectomy was performed. The patient was discharged at $24 \mathrm{~h}$ after intervention without any complications.

Conclusion: in the case of primary hyperaldosteronism, surgery implies a possibility near $100 \%$ of controlling hypertension with medication and a possibility of cure of between $30-60 \%$. Laparosco-pic acces is nowadays the gold standard. 


\section{Resumen}

Introducción: los adenomas suprarrenales son tumores benignos que pueden tener o no hiperactividad (producción hormonal). El síndrome de Conn es una causa de HTA secundaria debida a una producción excesiva de aldosterona que genera un hiperaldosteronismo primario.

Presentación del Caso clínico: mujer de 52 años con antecedente de HTA de 4 años de evolución con mal control a pesar de medicación en los últimos 2 meses. Ante la refractariedad se indicó estudio de sospecha hipertensión secundaria/refractaria: Analíticamente se constató niveles elevados de Aldosterona que sugieren diagnóstico de hiperaldosteronismo primario. El TC evidenció una masa a nivel suprarrenal derecho. Ante esta sospecha se realizó una suprarrenalectomía derecha laparoscópica. Postoperatorio sin incidencias, siendo dada de alta a las $24 \mathrm{~h}$.

Conclusión: en caso de hiperaldosteronismo primario la cirugía supone una posibilidad de control de la HTA con medicación cercana al 100\% y de curación completa del 30-60\%. El acceso laparoscópico es la vía de elección actualmente.

\section{Keywords}

Primary Hyperaldosteronism, Conn`S Syndrome, Adrenalectomy

\section{Introducción}

Se estima que en torno al 4\% de la población tiene tumoraciones adrenales, de las cuales un $85 \%$ son no funcionantes y un $15 \%$ son funcionantes, incluyendo adenomas funcionantes y feocromocitomas [1]. Los adenomas suprarrenales son tumores benignos, de pequeño tamaño, localizados en la corte-za suprarrenal y casi siempre únicos. Pueden tener o no actividad dando lugar a cuadros clínicos y bioquímicos de hiperfunción cortical, siendo más frecuentemente no funcionantes.

La gran mayoría de casos de Hipertensión son primarios, pero ocasionalmente es secundaria a en-fermedades subyacentes. La prevalencia de HTA en España oscila en torno al 35\% en la población adulta, siendo la proporción de hipertensión secundaria inferior 5\% [2]. La hipertensión arterial se-cundaria es más común en individuos menores de 30 años y es con mayor frecuencia refractaria al tratamiento habitual. El interés en el diagnóstico de esta entidad radica en la evidencia de que se acompaña de mayor mortalidad y que la persistente elevación tensional acaba provocando alteracio-nes estructurales del sistema cardiocirculatorio [3]. La mayor parte son de causa renovascular; la etiología endocrina es poco frecuente, el hiperaldosteronismo primario sólo ocupa un 5-15\% [4]. El síndrome de Conn o hiperaldosteronismo primario, corresponde a $0.05-2.2 \%$ de la población global de hipertensos y es una de las pocas causas potencialmente curables de hipertensión [5]. El objetivo del presente caso es subrayar la importancia del estudio de todo paciente con sospecha de hiperten-sión secundaria, hipertensión refractaria a tratamiento y/o con alteraciones hidroelectrolíticas asociadas.

\section{Caso clínico}

Presentamos el caso de una mujer de 52 años sin antecedentes de interés con diagnóstico de hiper-tensión esencial de 4 años de evolución en tratamiento con enalapril y amlodipino. Consulta por mal control tensional en los últimos dos meses asociado a cefalea occipital y parestesias ocasionales en miembros inferiores, a pesar del adecuado cumplimiento del tratamiento pautado y de las medidas higiénico-dietéticas habituales.

Ante la refractariedad del tratamiento hipotensor se realiza estudio para evaluar causas de hiperten-sión secundaria.

En la exploración física, la paciente presentaba buen estado general con PA 205/100mmHg, 
FC: $701 \mathrm{mp}$. Peso $67 \mathrm{~kg}$, talla $172 \mathrm{~cm}$. Auscultación cardiopulmonar sin interés y abdomen sin hallazgos. En el hemograma destaca normonatremia e hipocaliemia discreta $(7,1 \mathrm{mg} / \mathrm{dl})$, no atribuible a diuréticos que no incluía su tratamiento habitual. El nivel plasmático de aldosterona fue de $410 \mathrm{pcg} / \mathrm{ml}(40-300 \mathrm{pcg} / \mathrm{ml})$, con un nivel renina plasmaticade $0,81 \mathrm{ng} / \mathrm{ml} / \mathrm{h} \quad(0,6-4,3 \mathrm{ng} /$ $\mathrm{ml} / \mathrm{h}$ ), con lo cual se consideró la posibilidad de hiperaldosteronismo primario y se solicitó un $\mathrm{TC}$ de abdomen en el que se descubre un tumor suprarrenal derecho de $2.5 \mathrm{~cm}$ de diámetro.

Ante el diagnóstico de sospecha de adenoma suprarrenal derecho productor de Aldosterona se consideró a la paciente candidata a tratamiento quirúrgico y se realizó una suprarrenalectomía dere-cha laparoscópica con la paciente en posición de decúbito lateral izquierdo con brazo derecho sobre reposabrazo. Postoperatorio sin incidencias siendo dado de alta a las $24 \mathrm{~h}$. El estudio anatomopato-lógico confirmó el diagnóstico de adenoma de corteza suprarrenal de $1,8 \mathrm{~cm}$ de diámetro.

En el control ambulatorio se halló mejoría de las cifras tensionales, actualmente sin necesidad de tratamiento médico a los 6 meses del postoperatorio.

\section{Discusión}

El síndrome de Conn, también conocido como hiperaldosteronismo primario, es una de las causas conocidas de HTA secundaria. La HTA es debida a una producción excesiva de aldosterona que a nivel renal induce un aumento en la reabsorción de sodio y agua, lo que se traduce en un incremento del volumen intravascular y, secundariamente, en una elevación de la presión arterial [6]. Se diag-nostica con más frecuencia a la edad de 30 a 60 años, con predominio en el género femenino, con una relación 1.5:1 [7]. En muchas ocasiones es completamente asintomático y la única forma de pre-sentación es una hipertensión refractaria. Cuando se producen síntomas suelen ser secundarios a los trastornos hidroelectrolíticos asociados, fundamentalmente hipocalemia e hipernatremia. La descrip-ción clásica era hipertensión e hipopotasemia pero actualmente más de la mitad de casos diagnosticados no tienen hipopotasemia. Además estos pacientes tienen un aumento de complicaciones car-diovasculares mayor que el de la hipertensión primaria de origen no aclarado.

De acuerdo a la guía clínica de la sociedad Endocrina Europea de 2016, se recomienda el estudio de aldosteronismo primario en pacientes con [8]:

- Hipertension e hipocaliemia espontánea a o con bajas dosis de duirético.

- Hipertensión severa $(>150 \mathrm{mmHg}$ de sistólica o $>100 \mathrm{mmHg}$ de diastólica) o hipertension refracta-ria (definida como hipertensión mal controlada con 3 fármacos antihipertensicos que incluyan un antiadrenérgico, un agente vasodilatador y un diurético)

- Hipertensión con un incidentaloma suprarrenal

- Hipertensión y apnea del sueño

- Hipertensión e historia familiar de hipertensión o accidente cerebrovascular a una edad temprana ( $<40$ años)

- Todos los familiares de primera generacion de pacientes con aldosteronismo primario

El diagnóstico se basa en los niveles de aldosterona y renina en plasma. Los individuos con aldoste-ronismo primario tienen concentraciones plasmáticas típicas de aldosterona $>15 \mathrm{ng} / \mathrm{dL}$ ( $>0,42 \mathrm{nmol} / \mathrm{L}$ ) y niveles bajos de actividad plasmática de la renina. La prueba de imagen de elección es el TAC y resonancia magnética. Las imágenes ayudan a establecer si el hiperaldosteronismo es idio-pático o secundario a adenoma y si éste es unilateral o bilateral.

Se han descrito diversas causas de hiperaldosteronismo, siendo las más comunes los adenomas productores de aldosterona $(30-40 \%)$ y el hiperaldosteronismo bilateral idiopático (60-70\%). El tratamiento de elección para el adenoma es la suprarrenalectomía laparoscópica, logrando el control de la tensión arterial posterior a la resección, incluso sin 
necesidad de tratamiento hipotensor en un 30$60 \%$ [9]. Preoperatoriamente se recomienda evaluar la función cardiaca y renal. Mantener el tratamiento hipo-tensor habitual y dieta hiposódica. Se considera curación las siguientes situaciones: 1) disminución de la presión sistólica menor de $140 \mathrm{mmHg}$ y presión diastólica menor de $90 \mathrm{mmHg}$ sin la ingesta de antihipertensivos, y 2) si la presión arterial disminuye $20 \mathrm{mmHg}$ y el consumo de fármacos disminuye [10].

Se han propuesto una seria de predictores para evaluar aquellos pacientes con mayor probabilidad de curación tras la suprarrenalectomía. Consiste en 4 características clínicas: tratamiento antihipertensi-vo con 2 o menos fármacos (2 puntos), IMC $\leq 25 \mathrm{~kg} / \mathrm{m} 2$ (1 punto), duración de HTA menor a 6 años (1 punto), sexo femenino (1 punto) [11]. Otros factores asociados con menor intensidad: ausencia de historia familiar de HTA, edad menor, mayor cociente preoperatorio de aldosterona/renina plasmáti-cas. Basándonos en dichos predictores, nuestra paciente de sexo femenino con un IMC de 22, dura-ción de HTA de $<$ de 6 años y estar en tratamiento con 2 fármacos, tiene probabilidad alta de reso-lución quirúrgica de la HTA. De hecho, nuestra paciente abandonó la medicación antihipertensiva a los 6 meses de la cirugía.

\section{Conclusiones}

En caso de hiperaldosteronismo primario, la cirugía supone una posibilidad de control adecuado con tratamiento antihipertensivo cercana al $100 \%$ y de curación del 30-60\%, reduciendo la morbimorta-lidad asociada a la HTA. La cirugía laparoscópica es hoy en día la técnica de elección en esta pato-logía, con beneficios postoperatorios como mejor control del dolor y menor estancia hospitalaria.

\section{Declaración de conflicto de interés}

No hay conflictos de interés conocidos asociados con esta publicación y no ha habido apoyo financiero para este trabajo que pudiera haber influido en el resultado. La presente investigación no ha recibido ninguna beca específica de agencias de los sectores público, comercial o sin ánimo de lucro.

\section{Bibliografía}

1. Bovio S, Cataldi A, Reimondo G, Sperone P, Novello S, Berruti A, Borasio P, Fava C, Dogliotti L, Scagliotti GV, Angeli A, Terzolo M: Prevalence of adrenal incidentaloma in a contemporary computerized tomography series. J Endocrinol Invest. 2006; 29: 298-302.

2. V. Giner Galvañ, M.J. Esteban Giner. Estrategias para el despistaje de la HTA secundaria. Hipertensión (Madr), 23 (2006): 284-297.

3. Williams B, Mancia G, Spiering W, Agabiti Rosei E, Azizi M, Burnier M, et al. 2018 ESC/ ESH guidelines for the management of arterial hypertension: The task force for the management of arte-rial hypertension of the European Society of Cardiology and the European Society of Hypertension. J Hypertens. 2018;36(10):19532041.

4. Monticone S, Burrello J, Tizzani D, Bertello C, Viola A, Buffolo F, et al. Prevalence and clinical manifestations of primary aldosteronism encountered in primary care practice. J Am Coll Cardiol. 2017 Apr 11;69(14):1811-1820.

5. RP.M. Kearney, M. Whelton, K. Reynolds, P. Muntner, P.K. Whelton, J. He. Global burden of hypertension: Analysis of worldwide data. Lancet. 2005; 365: 217-223.

6. J. Chirino Romo, I. Wiener-Carrillo, B.M. Cervantes-Valladolid, et al. Hipertensión arterial se-cundaria a síndrome de Conn., 9 (2011), pp. 221-224.

7. J. Padilla-Piña, J. Arriaga-Aguilar, R.A. García-Vásquez, A. Razo-Garcia, F.E. GarciaMartinez, et al. Síndrome de Conn y su manejo laparoscópico. Revista mexicana de urología. 2016; 76 (4): 261-264.

8. Funder JW, Carey RM, Mantero F, Murad MH, Reincke M, Shibata H, et al. The management of primary aldosteronism: Case detection, diagnosis and treatment: an endocrine society clinical practi-ce guideline. The journal of clinical endocrinology \& Metabolism. 2016: 101(5): 1889-1916.

9. Karina Sifontes, Manuel Heras, Daniel Puentes, Pablo Vázquez, María Astrid Rodríguez et al. Hipertensión arterial persistente y fracaso 
renal agudo tras suprarrenalectomía por adenoma. 11. R. Zarnegar, W.F. Young Jr., J. Lee, M.P. Diálisis y trasplante. 2014; 35 (3): 115- 118. Sweet, E. Kebebew, D.R. Farley, et al.

10. B. Edwin, I. Raeder, E. Trondsen, et al. The aldosteronoma resolution score: Predicting Outpatient laparoscopic adrenalectomy in complete resolution of hypertension after patients with Conn's syndrome. Surg Endosc. adrenalec-tomy for aldosteronoma. Ann Surg. 2001; 15: 589-591. 2008; 247: 511-518. 